\title{
Gold-Cobalt Electrodeposits
}

\section{MICROSTRUCTURE AND SURFACE TOPOGRAPHY}

\author{
S. J. Harris and E. C. Darby \\ Department of Metallurgy and Materials Science, University of Nottingham, England
}

\begin{abstract}
Alloy gold deposits which are used widely as contact materials in the electronics industry are shown to have complex microstructures. The existence of ultra-fine grain sizes and polymer inclusions may be responsible for the improved wear behaviour of these coatings.
\end{abstract}

Gold of high purity electroplated on to contact surfaces causes problems of adhesion and rapid wear, and it has been necessary to develop alloys that maintain satisfactory electrical performance while minimising mechanical damage; gold-cobalt and gold-nickel systems are used widely in the electronics industry. In the case of gold-cobalt, deposits have been produced with up to 0.2 per cent cobalt from an acid citrate bath to which gold and cobalt ions have been added as gold potassium cyanide and cobaltous sulphate respectively.

The reasons why the wear resistance of such deposits is improved are quite complex and many authors (1-5) have postulated theories. Some inferences have been drawn from chemical analysis data $(3,4)$ and these indicate a strong relationship between contact performance and either potassium, cobalt or carbon contents. Investigators $(1,2,5,6)$ have attempted to assess structure with mechanical and electrical behaviour, with some success.

At the maximum resolving power of an optical microscope it is impossible to reveal meaningful features on a polished and etched section taken from a gold-cobalt deposit, and to resolve grain structure it is necessary to resort to transmission electron microscopy (T.E.M.). This requires thinning the material from $5 \mu \mathrm{m}$ to $\sim 0.1 \mu \mathrm{m}$ and argon ion bombardment was employed, after which the specimen was supported in an electron microscope operated at $100 \mathrm{kV}$.

A deposit produced at a current density of 0.5 $\mathrm{A} / \mathrm{dm}^{2}$ was examined and this showed an ultrafine structure, shown in Figure la. On making measurements of grain diameter it was found that a limited range of values was present, i.e. 100 to $300 \AA$, with a mean value in the neighbourhood of $170 \AA$. When the instrument was changed to an electron diffraction mode the resultant pattern, shown in Figure $1 b$, was of a spotty ring type, showing that because of the fine grains, single crystal electron diffraction patterns were impossible to obtain. Within the grains a number of markings can be resolved, such as parallel lines running across the grains which may be interpreted as growth twins. In all electrodeposits of the common f.c.c. metals-copper, aluminium, nickel, etc.-growth twins have often been observed in very high densities, and gold with its low stacking fault energy would not be expected to be otherwise.

At intergranular and intragranular sites a number of voids are to be seen in the bright field micrograph. This feature has a mean diameter of $\sim 75 \AA$ and if assumptions were made about the thickness of the foil it has been calculated that void number was in the neighbourhood of $10^{16} / \mathrm{cm}^{3}$. These voids could have arisen from two sources: (i) co-deposition of hydrogen in a process which is $\sim 80$ per cent efficient with respect to gold deposition, or (ii) damage caused by the argon ion beam during preparation. Of these possibilities source (i) is considered to be the most likely. No evidence could be found of second phase particles or precipitates in bright field observations.

In foils prepared from deposits that had been kept for lengthy periods since production, a different type of structure was observed in some regions. The micrograph of Figure 2a shows a fibrous form with each "fibre" having a diameter of $\sim 250 \AA$. Electron diffraction patterns taken in this region show diffuse rings indicating an amorphous structure as in Figure 2b. In between these groups of "amorphous fibres", patches of fine grained gold can be observed. These regions have been interpreted as "polymeric material" which has been formed during electrodeposition and may have undergone changes under oxidising conditions during storage. Such a material has been found by a number of investigators $(4,5)$ as a thin cracked film when the gold is dissolved away in aqua regia, and analysis of the film has indicated that it is some form of cyanide polymer. Although it appears in a fibrous form in Figure 2a it may be that this morphology resulted from a rumpling action on the film-like form during preparation in the argon ion beam. 

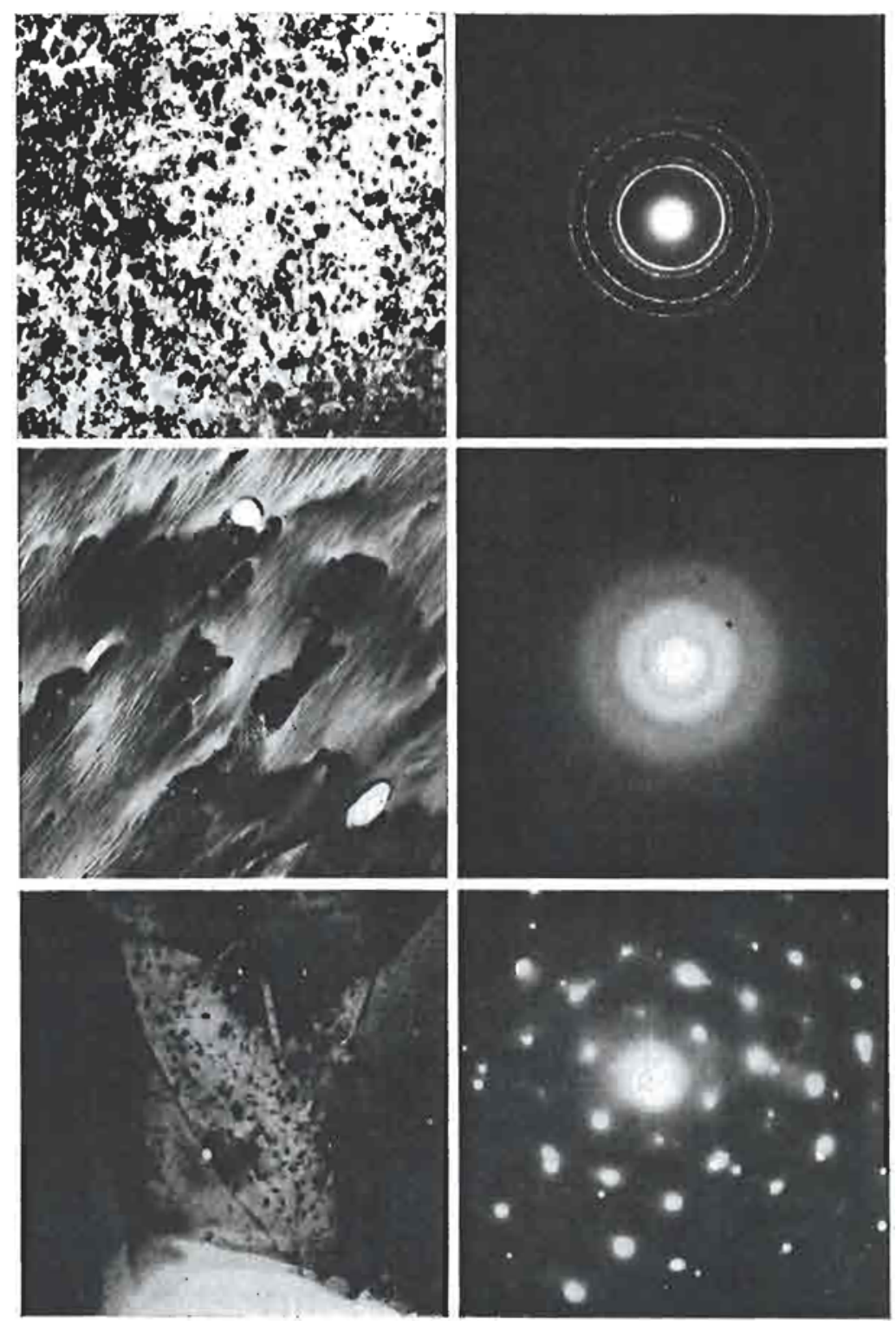

Fig. Ia (top, far left) Trangmission eleetron micrograph of as-deposited gold-cobalt alloy showing the presence of growth twins and voids $\times 100 \mathrm{k}$

Fig. 1b (top) "Spotty ring" electron diffraction pattern obtained in conjune. tion witl Fig. Ia

Fig. 2a (middle, far left) Transmission electron micrograph of a deposit containing "fibrous polymer" regions $\times 50 k$

Fig. 2b (middle) Electron diffraction pattern showing diffuse rings taken in conjunction with Fig. $2 a$

Fig. 3a (bottom, far left) Micrograph of a deposit annealed for 1 hour at $400^{\circ} \mathrm{C}$, quenched and aged at $20^{\circ} \mathrm{C}$ for 480 hours, showing large grain size and twins and a number of strain lobes $\times 100 \mathrm{k}$

Fig. 3b (bottom) Electron diffraction patteri from a selected area of foil shown in Fig. 3a, showing a single crystal diffraction pattern and weak "streaking" which may be due to precipitation

thus indicating a possible relationship between polymer and cobalt distributions in the gold. Hence, a composite structure involving fine grained gold, "precipitation hardened" with voids and cobalt which contains pockets of a cyanide polymer appears to be evolving from these observations. From an electrical contact standpoint the hardening of the gold should increase its wear resistance while the presence of other impurities such as polymer films will tend to reduce

From these T.E.M. observations it is clear that gold-cobalt deposits are complex materials. The microhardness of the deposits has been measured in the range 160 to $220 \mu \mathrm{HV}_{20 g}$, the actual values depending on both composition and operating conditions. These values are well above those achieved in pure gold deposits ( 40 to $80 \mu \mathrm{HV}_{20}$ ) and the major component of this change must be associated with the extremely fine grain size which will significantly raise the effective yield stress of the material. More recently published work (5-7) indicates that the grain size may not be the only contribution to this large change in hardness but other components such as cobalt precipitation, together with void number and distribution, could affect the issue. The presence of the polymer can account for the increased carbon content of the deposits and some workers (3) have noted that there is a correlation between cobalt and carbon content, the ability for surfaces to weld together; on the other hand these films will tend to increase the contact resistance. It would seem that some delicate balance is achieved between these two latter factors in as-deposited contact surfaces.

During the fabrication of reed relays, parts of the electrodeposit can be heated for short periods to between 400 and $500^{\circ} \mathrm{C}$, while in service they may operate at temperatures up to $200^{\circ} \mathrm{C}$. Transmission electron micrographs have been taken (7) of thinned gold deposits stripped from substrates before heat treatment in the temperature range 20 to $600^{\circ} \mathrm{C}$. Samples treated at $400^{\circ} \mathrm{C}$ provide a good example of the significant changes which have taken place in microstructure; Figure 3a shows that the grain size has increased by approximately twenty times to $0.5 \mu \mathrm{m}$ and the void size by four times to $400 \AA$. Instead of the spotty ring diffraction pattern, a single crystal pattern, Figure $3 b$, is now achieved, 
Fig. 4 Scanning electron micrographs of the surface of a gold-cobalt deposit after annealing for 1 hour at $400^{\circ} \mathrm{C}$ Fig. 4a (top) $\times 40$, Fig. 4 b (bottom) $\times \mathbf{4 0 0 0}$

which can be indexed as gold. Within the gold grains of Figure 3a there is some evidence of strained regions in the lattice-strain lobes, which in turn may be interpreted as precipitates of cobalt. These could have resulted from two sources: (i) dissolution of cobalt at $400^{\circ} \mathrm{C}$ and reprecipitation at lower temperatures, or (ii) coarsening of unresolved cobalt clusters in the as-deposited state. Further evidence which supports the existence of cobalt as a separate phase comes from the streaks observed in the electron diffraction pattern.

Polymer regions could be still observed in the foils after treatment at $400^{\circ} \mathrm{C}$, although their morphology had changed from fibrous to cellular. It has also been noted (7) that the change brought about in the grain size by heat treatment has an effect on the microhardness, which has fallen to $\sim 60 \mu \mathrm{HV}_{20}$. Deposits so treated would be expected to have significantly poorer wear resistance.

The surface of as-deposited gold-cobalt is extremely smooth and almost featureless when examined at a magnification of $\times 5000$ in the scanning electron microscope. However, when the deposits are heat treated changes begin to occur as the annealing temperature approaches $300^{\circ} \mathrm{C}$. Examples of the changes in surface topography after treatment for one hour at $400^{\circ} \mathrm{C}$ are shown in Figure 4. At low magnifications, a series of ripples is resolved ( $60 \mu \mathrm{m}$ in width) evenly spaced over the whole surface. Distributed on the rippled peaks and valleys are numerous small apparently white features. Examination at higher magnifications resolve these features as hemispherical bubbles, each $\sim 15 \mu \mathrm{m}$ in diameter. Increasing the magnification still further reveals a series of small pores over the whole surface; these pores have a diameter of $\sim 0.3 \mu \mathrm{m}$ (Figure $4 b)$. Also present are a number of surface protrusions of similar dimensions to the pores. Increasing the temperature of the annealing treatment above $400^{\circ} \mathrm{C}$ results in an increase in pore and protrusion sizes, together with an increase in the incidence of loose debris.

The degradation of the surface that takes place during this treatment may result from a number of sources: (i) relief of stress, (ii) expansion of gas, e.g. hydrogen, or from water occluded during electrodeposition, (iii) decomposition of the cyanide polymer and (iv) oxidation of the base metal component. On the basis of observations taken over the whole temperature range and relating wherever possible to the available insight


into internal microstructure of the deposits, it seems more likely that the major changes in surface topography have come about because of polymer decomposition. If damage of the kind indicated begins to take place during fabrication or after long periods in service then the effective surface area of contact will decrease appreciably and thus contact resistance will increase rapidly.

On the basis of microstructural and chemical analyses a pattern is emerging which is beginning to provide clearer explanations of the complexity of this technologically important group of electrodeposited gold alloys. Furthermore, clearer guidelines to the conditions of involvement in fabrication processes and usage are coming about which could help reduce the problems of service failure.

\section{References}

1 A. J. Solomon and M. Antler, Plating, 1970, 57, (8), 812

2 M. Antler, Plating, 1973, 60, (5), 468

3 L. Holt, R. J. Ellis and J. Stanyer, Plating, 1973, 60, (9), 910 and 918

4 G. B. Munier, Plating, 1969, 56, (10), 1151

5 E. C. Darby and S. J. Harris, Trans. Inst. Metal Finish., 1975, 53, (3), 138

6 P. S. Willcox and J. R. Cady, Plating, 1974, 61, (12), 1117

7 S. J. Harris, E. C. Darby, K. Bridger and A. E. Mason, to be published 